\title{
Use of Nomograms for Early Detection in Prostate Cancer
}

\author{
Devon C. Snow, MD, and Eric A. Klein, MD, Cleveland, Ohio
}

\begin{abstract}
Key Words
Nomogram, prostate cancer, risk calculator, screening tools

Abstract

Since the first introduction of prostate-specific antigen (PSA) as a screening tool in the 1980s, the accurate diagnoses of clinically significant prostate cancer remains a challenge. Analysis of a correlation between PSA levels and prostate biopsies of men with PSA $3 \mathrm{ng} / \mathrm{mL}$ or less in the placebo group of the Prostate Cancer Prevention Trial suggested that no "normal" PSA level exists. With the acknowledgement that PSA level is considered a continuum rather than a dichotomous marker, accurately diagnosing clinically significant prostate cancer is even more challenging. Nomograms are increasingly being used as tools in the clinical setting to address this challenge. Through incorporating multiple clinical factors, such as PSA, digital rectal examination, age, race, prostate volume, family history, and previous negative biopsy, risk calculators can improve sensitivity of diagnosis over using a PSA cutoff alone. This article discusses the rational for the use of nomograms and the advantages and limitations for the most commonly used nomograms. (JNCCN 2010;8:271-276)
\end{abstract}

In 2009 alone, an estimated 192,280 men will be diagnosed with and 27,360 will die of prostate cancer, ${ }^{1}$ making it the most common cancer diagnosed in men after skin cancer and the second leading cause of cancer death. Although no cure is available for metastatic prostate cancer, treatment can be curative if it is caught early. The goal of early diagnosis and radical treatment was first suggested in $1905 .^{2}$ The introduction of

From the Glickman Urological and Kidney Institute, Cleveland Clinic Lerner College of Medicine, Cleveland, Ohio.

Submitted October 10, 2009; accepted for publication December 7, 2009.

The authors have disclosed that they have no financial interests, arrangements, or affiliations with the manufacturers of any products discussed in the article or their competitors.

Correspondence: Eric A. Klein, MD, Glickman Urological and Kidney Institute, Cleveland Clinic Lerner College of Medicine, Desk Q10-1, 9500 Euclid Avenue, Cleveland, OH 44195. E-mail: kleine@ccf.org prostate-specific antigen (PSA) as a screening tool in the late 1980s induced both a clinical and pathologic stage migration that is believed to have contributed at least partly to the observed reduction in prostate cancer mortality since $1990 .^{3}$

The hope was that the Prostate, Lung, Colorectal, and Ovarian (PLCO) Cancer Screening Trial in the United States ${ }^{4}$ and the European Randomized Study of Screening for Prostate Cancer (ERSPC) would definitely answer the question of whether screening impacts prostate cancer mortality. ${ }^{5}$ Unfortunately, the trials came to opposite conclusions; the PLCO showed no mortality benefit when an annual screening PSA of $4 \mathrm{ng} / \mathrm{mL}$ or greater or an abnormal digital rectal exam (DRE) triggered biopsy for diagnosis, whereas the ERSPC showed a 20\% reduction in prostate cancer-specific mortality. Many factors, including a high rate of prior screening, high drop-in rate, and delayed biopsies in the PLCO contributed to the differing results. At best the ERSPC shows that screening can reduce mortality by a modest degree.

Although PSA has been traditionally considered a dichotomous marker for prostate cancer risk, data from the placebo arm of the Prostate Cancer Prevention Trial (PCPT) challenge this notion. In this trial, men at average risk for prostate cancer (PSA $<3 \mathrm{ng} / \mathrm{mL}$ and normal DRE) were either treated with finasteride or placebo and followed up for 7 years, with biopsy performed either "for cause" (rise in PSA or suspicious DRE) or at the end of the trial. ${ }^{6}$ Results found that $15 \%$ of men with a PSA less than 4 were diagnosed with prostate cancer on biopsy, with $2.3 \%$ of the cancers being highgrade or Gleason score 7 or greater. Most notably, results showed that there was no PSA below which the risk for cancer was zero and that no specific PSA level had sufficient sensitivity or specificity to be used as a diagnostic cutoff. ${ }^{7}$ However, the data also showed that a man 
with a PSA of $0.5 \mathrm{ng} / \mathrm{mL}$ has a different risk than one with a PSA of $3.9 \mathrm{ng} / \mathrm{mL}$. The challenge is how to produce tools that capture all of the information contained in PSA levels and that can be used for counseling patients.

Factors other than PSA affect the risk for having prostate cancer and may be useful for risk stratification, including PSA velocity, PSA density, DRE, use of finasteride, age, race, family history of prostate cancer, and history of previous negative biopsy. In this discussion nomograms and risk calculators are essentially interchangeable terms. Nomograms are developed by analyzing large population data, thereby capturing the impact of all of these factors and allowing the prediction of individual risk. Nomograms can easily calculate the risk for linear, such as PSA, and categorical parameters, such as race, into a percentage risk that helps facilitate counseling discussions.

The advantages of nomograms are that the combination of multiple factors, each with an independent predictive value, can improve the accuracy of the prediction of prostate cancer risk over any one factor alone. Furthermore, the statistical models that underlie these tools can capture all of the information contained in a continuous variable. Additional advantages include the ability to validate the models in other cohorts to maximize generalizability and the ability to add new variables, including biologic markers, to the calculator as they are validated.

Another advantage of nomograms is that they eliminate the subjective bias of clinician estimates and provide a single prediction of risk to the patient. A challenge with this approach, however, is determining what positive predictive value is an acceptable threshold for biopsy. If prevailing wisdom suggests that a biopsy is indicated at a $25 \%$ risk, would a patient be comfortable with avoiding biopsy if he discovers he has a $24 \%$ risk of cancer? It is possible that nomograms, although improving accuracy of predictions, may also increase the percentage of patients getting biopsies and therefore may diagnose clinically insignificant prostate cancer. This effect may be tempered if available nomograms presented their risks as a risk range, given that nomograms are not $100 \%$ accurate. Typically nomograms aim for a total predictive accuracy greater than $70 \%$ to $80 \%$ to be valid. ${ }^{8}$ A downside of presenting risk as a range is that patients may have difficulty interpreting ranges, and may either avoid biopsy if the range included zero, or aggressive- ly opt for biopsy for any risk above zero, potentially increasing the likelihood of diagnosing biologically insignificant cancer.

Two primary nonexclusive methods to evaluate the accuracy of nomograms are generally used. One is to evaluate the area under the curve (AUC) of the receiver operating characteristic (ROC). The ROC is a graphical plot of sensitivity versus specificity. A $100 \%$ accurate nomogram would yield a single point representing $100 \%$ sensitivity and $100 \%$ specificity (i.e., no false-positives or -negatives). This location at the upper left-hand side of a ROC curve is also called the perfect classification. Random predictions should theoretically be a $y=x$ line or a diagonal line, which has been called the line of no discrimination. The AUC measures the area under ROC curves to create a single number to define accuracy. An ideal ROC would have an AUC of 1, whereas a random ROC would yield an AUC of 0.5. When predicting risk for an individual, a 95\% confidence interval can also be used to provide a descriptor of the accuracy of the nomogram that can be useful during individual calculation. Recently a proliferation of predictive tools has attempted to improve the accuracy at which prostate cancer risk can be predicted.

This proliferation of nomograms brings to head the need for clinicians to critically analyze the available tools. When analyzing the current literature, the specific limitations of the proposed risk calculator must be considered (i.e., small cohort size, narrow cohort characteristics, or even the lack of multivariate analysis of all currently known risk factors for prostate cancer). The limitations of risk calculators in general are also important to acknowledge. To be valid, patients using the tool must have similar features to the cohort that was used to develop the nomogram. In addition, novel biomarkers of prostate cancer not available at cohort analysis for nomogram development may not be available as variables. Likewise, newly identified risk factors, such as lifestyle or obesity, will not be incorporated into available nomograms unless that information was available in the population used to develop the initial tool.

Unless a tool predicts high- versus low-grade cancer risk, overdiagnosis of low-grade cancers will continue. As clinical practice changes, previously developed nomograms will become invalid. For example, DRE is highly subjective, and as physicians rely increasingly on PSA for screening the sensitivity of 
DRE may change. If this occurs, then patients with abnormal DREs 10 years ago are unlikely to be categorically similar to ones whose risk is calculated today. And finally, although a large amount of literature exists on the development of different nomograms, only survey data are available on their clinical use. ${ }^{9}$ Even though decision aids, including prediction models, have been shown to impact patient's decision-making behavior, how nomograms change clinical outcomes is entirely unproven. ${ }^{10}$

Although this paper focuses on reviewing the use of nomograms in the detection of prostate cancer, many other types of predictive tools are currently being developed, including risk groupings, artificial neural networks, probability tables, and classification and regression tree (CART) analysis. Artificial neural networks can be described as a computational model that is an adaptive system that changes structure based on a learning phase and is best used to model very complex relationships with unclear patterns among the data. Risk grouping includes assigning patients with prostate cancer into homogenous groups and predicting risk for similar future patients based on the findings of the previous risk group. Probability tables such as Partin staging tables will use multiple variables but will typically change linear data into categorical data sets. CART analysis take different inputs and then create a decision tree. Herein the characteristics, advantages, and limitations of the most commonly used nomograms to predict the presence of prostate cancer in clinically practice are described (Table 1).

\section{PCPT Risk Calculator}

The PCPT risk calculator was developed from analyzing men in the placebo arm of the PCPT. ${ }^{7,11}$ This was a phase III randomized, double-blind, placebocontrolled trial comparing the rates of cancer in men taking finasteride versus placebo for 7 years. The placebo arm consisted of 5519 men with a PSA of

\section{Table 1 Comparison of Presented Risk Calculators}

\begin{tabular}{|c|c|c|c|c|c|}
\hline Study & Study Population & Variables & Strengths & Weaknesses & AUC \\
\hline PCPT & $\begin{array}{l}\text { - } 5519 \text { American men } \\
\text { - Age > } 55 \text { years } \\
\text { - Average age } 62 \text { years } \\
\text { - } 95.6 \% \text { white } \\
\text { - } 3.2 \% \text { African } \\
\text { American } \\
\text { - } 1.2 \% \text { other }\end{array}$ & $\begin{array}{l}\text { - PSA } \\
\text { - Age } \\
\text { - Race } \\
\text { - Family history } \\
\text { - DRE } \\
\text { - Prior prostate biopsy } \\
\text { - Use of finasteride } \\
\text { - Optional PCA3 }\end{array}$ & $\begin{array}{l}\text { - } 95 \% \text { Cls for risk } \\
\text { predictions } \\
\text { - High- and } \\
\text { low-grade risk } \\
\text { prediction } \\
\text { - Externally } \\
\text { validated }\end{array}$ & $\begin{array}{l}\text { - Low starting PSA } \\
\text { - Minimal lower urinary } \\
\text { tract symptoms } \\
\text { - No prostate volume } \\
\text { measurements }\end{array}$ & $\bullet 0.72$ \\
\hline ERSPC & $\begin{array}{l}\text { - } 8621 \text { Dutch men } \\
\text { - 55-74 years old }\end{array}$ & $\begin{array}{l}\text { - PSA } \\
\text { - Age } \\
\text { - Family history } \\
\text { - Prostate volume (DRE } \\
\text { or TRUS) } \\
\text { - IPSS }\end{array}$ & $\begin{array}{l}\text { - Use of prostate } \\
\text { volume }\end{array}$ & $\begin{array}{l}\text { - Different population } \\
\text { used to produce risk } \\
\text { calculator } \\
\text { - IPSS as presumptive } \\
\text { marker for TRUS } \\
\text { volume } \\
\text { - Does not include CI/ } \\
\text { range of risk } \\
\text { - Risk for cancer for PSA } \\
<3 \text { ng/mL based on } \\
\text { extrapolation }\end{array}$ & $\begin{array}{l}\text { - Not } \\
\text { calculated }\end{array}$ \\
\hline Sunnybrook & $\begin{array}{l}\text { - } 3108 \text { Canadian men } \\
\text { - Age > } 40 \text { years } \\
\text { - Average age } 62 \text { years } \\
\text { - } 82 \% \text { white } \\
\text { - } 9 \% \text { African American } \\
\text { - } 9 \% \text { other }\end{array}$ & $\begin{array}{l}\text { - PSA } \\
\text { - Age } \\
\text { - Race } \\
\text { - Family history } \\
\text { - Prostate volume } \\
\text { - DRE } \\
\text { - IPSS } \\
\text { - Free-to-total PSA ratio }\end{array}$ & $\begin{array}{l}\text { - Use of prostate } \\
\text { volume } \\
\text { - High- and } \\
\text { low-grade risk } \\
\text { prediction } \\
\text { - External validation } \\
\text { ongoing }\end{array}$ & $\begin{array}{l}\text { - Minority of patients } \\
\text { with PSA }<4 \mathrm{ng} / \mathrm{mL} \\
\text { - Majority white cohort } \\
\text { - Does not include } \mathrm{Cl} / \\
\text { range of risk }\end{array}$ & $\bullet 0.74$ \\
\hline
\end{tabular}

Abbreviations: AUC, area under curve; ERSPC, European Randomized Study of Screening for Prostate Cancer; DRE, digital rectal examination; IPSS, International Prostate Symptom Score; PCPT, Prostate Cancer Prevention Trial; PSA, prostate-specific antigen; TRUS, transrectal ultrasound. 
$3 \mathrm{ng} / \mathrm{mL}$ or less and who underwent PSA and DRE screening annually. They also underwent sextant biopsy if DRE was abnormal, for PSA greater than $4 \mathrm{ng} / \mathrm{mL}$, or at the end the 7 -year study. The results analyzed were those from men who had at least 1 PSA measurement and DRE in the year before biopsy and at least 2 PSA measurements performed during the 3 years before the prostate biopsy so that PSA velocity could be calculated.

The authors analyzed race, DRE, biopsy history, family history of prostate cancer, age, PSA, and PSA velocity within 3 years of biopsy. In this study population, family history (father, brother, or son with prostate cancer), PSA level, and presence of an abnormal DRE were independently associated with an increased risk for prostate cancer, whereas a previous negative biopsy was independently associated with a decreased risk. Interestingly, PSA velocity was highly associated with but not an independent predictor of prostate cancer, and therefore was not included in the final model. A separate analysis was performed to develop a risk calculator for high- (Gleason $\geq 7$ ) versus low-grade cancers. ${ }^{11}$ This nomogram is available online at http://deb.uthscsa.edu/URORiskCalc/ Pages/uroriskcalc.jsp.

Limitations of this study include the fact that patients had low starting PSA and minimal lower urinary tract symptoms, which meant that the findings have not been validated for patients with benign prostatic hypertrophy. This is especially important given that other studies, most notably the ERSPC trial, show that statistically significant prostate volume, as measured with transrectal ultrasound (TRUS), is predictive of cancer. ${ }^{12}$

Other limitations are primarily based on the study population, because the study overwhelmingly enrolled white, healthy, and well-educated men. The identification of race was a dichotomous factor in this study, with participants categorized as African American versus other, which is a limitation resulting from the minimal racial diversity of subjects enrolled. In addition, the AUC for this nomogram was only slightly higher than the in-sample AUC for PSA level alone: 0.72 versus 0.678 , respectively. ${ }^{13}$ This means that the independent risk factors (race, family history, abnormal DRE, and history of previous negative biopsies) provide only a slight improvement over PSA alone.

Advantages of this study include that it was a relatively large cohort including 5519 patients in a randomized controlled trial, which eliminates many potential biases. The online version includes calculations of the $95 \%$ confidence interval for the risk predictions, allowing individuals to assess accuracy in real terms. In addition, this nomogram includes the risk assessment for high- and low-grade cancers. An option now exists that incorporates PCA3 test results showing an AUC of 0.696 that was based on an analysis of 521 men undergoing PCA3 testing at 4 different American sites. ${ }^{14}$ Although the study participants were overwhelmingly white, the results were externally validated with a more diverse population when analyzed against the San Antonio Center of Biomarkers of Risk for Prostate Cancer (SABOR) participants. No differences in risk could be attributed to age, family history, DRE, or ethnicity in the SABOR cohort. When comparing ROC curves for PSA alone and PCPT risk scores in the SABOR cohort, the PCPT risk score was only marginally better and the difference was not statistically significant. When examined specifically for African Americans, however, the PCPT calculator showed improved sensitivity and specificity. ${ }^{15}$

\section{ERSPC Trial}

Kranse et al. ${ }^{12}$ created a nomogram to predict biopsy outcome by analyzing 8621 Dutch men between 55 and 74 years of age who were randomized to the screening arm of the ERSPC. No exclusion criteria were based on DRE or PSA levels. These men submitted an American Urologic Association (AUA) symptom score and then underwent 3 screening tests, including a PSA, TRUS, and DRE. They were offered a lateralized sextant biopsy if they had a PSA level of $4 \mathrm{ng} / \mathrm{mL}$ or greater and/or a suspicious finding on either TRUS or DRE.12 A risk calculator based on the ERSPC data is available online at www.prostatecancer-riskcalculator.com. The online risk calculator, unlike the article published by Kranse et al., ${ }^{12}$ is based on 6288 male participants with the same inclusion criteria as those analyzed in the published article.

One limitation of this study is that no further information is available on how the men included in the online version of the ERSPC risk calculator differ from those in the published study. A major limitation of this risk calculator is that no reference 
to accuracy is available online or included in the results. The online calculator does not use TRUS volume and instead incorporates AUA symptom score, a presumptive marker for prostate size. Previously published data have determined that TRUS size and AUA symptom score are only weakly correlated on linear regression analysis $(\mathrm{R}=.072) .{ }^{16}$ Another limitation is that the published version of the risk calculator does not calculate AUC but instead illustrates adequacy of the model by stating that $95 \%$ confidence intervals of the expected number of cancers were within the range of the observed number. In this study, the prevalence of prostate cancer was low $(5 \%)$, excluding prostate cancer found in patients with PSA less levels than 1. However, this could be because of biopsy technique and the variability of prostate size. Because of this, PSA level less than 3 predictions were incorporated into the risk calculator by extrapolating the logistic regression, and the risk calculator only predicts the result of a sextant biopsy. Both the published and online versions of this calculator do not delineate the risk for high- versus low-grade cancer, which theoretically increases the risk for overdiagnosis of clinically insignificant disease.

Advantages include the addition of TRUS and analysis of prostate volume as variables. In a separate study, hypoechoic lesions seen on TRUS in addition to PSA density calculations were shown to be independent predictors of prostate cancer. ${ }^{17}$ This study showed that PSA, prostate volume, DRE, and TRUS were all more important predictors of prostate cancer than age, race, family history, or even the presence of a previous negative biopsy. ${ }^{12}$ Given the importance noted of both prostate volume and TRUS findings, an advantage of this nomogram is the ability to incorporate these data into the prediction of likelihood or prostate cancer on biopsy.

\section{Sunnybrook Prostate Cancer Risk Calculator}

Nam et al. ${ }^{18}$ developed a tool based on data from 3108 Canadian men, 408 of which had PSA less than $4.0 \mathrm{ng} / \mathrm{mL}$. These men were analyzed by PSA, DRE, age, family history of prostate cancer, race, International Prostate Symptom Score, and free versus total PSA ratio. The AUC was determined to be 0.74 for all tumors and 0.77 for high-grade (Glea- son $\geq 7$ ) tumors compared with 0.62 to 0.69 for PSA alone, respectively. ${ }^{18}$ This tool is available online at http://sunnybrook.ca/content/?page=Focus_OCC_ Care_GenUri_PRisk_Calc.

One major limitation is that this study has a minority of patients in the normal PSA range and although the AUC for this subset is 0.74 , the confidence interval for this is wide (0.63-0.86) and overlaps the one for PSA alone. Given more patients, this may become more accurate, but currently the nomogram is less accurate for patients with PSA less than $4 \mathrm{ng} / \mathrm{mL}$. Like the PCPT nomogram, this does not include an analysis of prostate volume because this was not measured in the cohort. This cohort had only a minority of different ethnicities with $82 \%$ white, 9\% Asian, and 9\% black. The impact of a previously negative biopsy was not evaluated. Other limitations are that the online version does not include an indication of the range of risk for each individual patient based on the accuracy of the nomogram.

A strength of this study is that it is being further validated with an analysis of 5000 additional Canadian patients with PSA greater than $4 \mathrm{ng} / \mathrm{mL}$ and 1000 with PSA less than $4 \mathrm{ng} / \mathrm{mL}$. In addition to having plans for further external validation, this nomogram also delineates the likelihood of highgrade (Gleason $\geq 7$ ) cancer, as well as the risk for any cancer.

\section{Conclusions}

The most fundamental reason to use a nomogram is to improve accuracy beyond using PSA cutoff alone. Schroder and Kattan ${ }^{19}$ looked at the predictive tools available in review of the literature and found 12 studies showing improvements in AUC greater than PSA (range, 0.02-0.26), 5 of which had external validation. The ones presented earlier are those that are easily available online and most commonly used.

There is much excitement about the possible addition of genetic and novel biochemical markers to risk calculators. One biomarker, PCA3, has already been incorporated into the PCPT risk calculator. ${ }^{14}$ Early prostate cancer antigen, although initially interesting, is unlikely to be clinically useful until further validation can be obtained, given the current controversies. ${ }^{20}$ Several susceptibility loci have been identified that, when validated, might add to the di- 
agnostic accuracy of standard variables. ${ }^{21}$

Even if a risk calculator were available that provided $100 \%$ accuracy, opportunities would still exist to improve diagnosis and treatment further downstream. For example, many risk calculators suffer from the fact that biopsy is an imperfect tool. One autopsy study reported that up to $24 \%$ of cancers can be missed on sextant biopsy. ${ }^{22}$ Furthermore, there is much room for improvement in delineating clinically significant versus insignificant prostate cancers. As the results of the ERSPC and PLCO trials show, diagnosing more patients with prostate cancer could lead to overtreatment with no effect on mortality.

\section{References}

1. Horner MJ, Ries LAG, Krapcho M, et al. SEER Cancer Statistics Review. 1975-2006. Available at: http://seer.cancer.gov/ csr/1975_2003/. Last accessed January 12, 2010.

2. Young HH. The early diagnosis and radical cure of carcinoma of the prostate: being a study of 40 cases and presentation of a radical operation which was carried out in four cases. Bull Johns Hopkins Hops 1905;16:315-321; reprinted in J Urol 2002;167:939-947.

3. Jhaveri FM, Klein EA, Kupelian PA, et al. Declining rates of extracapsular extension after radical prostatectomy: evidence for continued stage migration. J Clin Oncol 1999;17:3167-3172.

4. Andriole GL, Crawford ED, Grubb RL III, et al. Mortality results from a randomized prostate-cancer screening trial. N Engl J Med 2009;360:1310-1319. Erratum in: N Engl J Med 2009;360:1797.

5. Schröder FH, Hugosson J, Roobol MJ, et al. Screening and prostate-cancer mortality in a randomized European study. N Engl J Med 2009;360:1320-1328.

6. Thompson IM, Goodman PJ, Tangen CM, et al. The influence of finasteride on the development of prostate cancer. N Engl J Med 2003;349:215-224.

7. Thompson IM, Pauler DK, Goodman PJ, et al. Prevalence of prostate cancer among men with a prostate specific antigen level < or $=4.0$ ng per milliliter. N Engl J Med 2004;350:2239-2246.
8. Chun FK, Karakiewicz PI, Briganti A, et al. Prostate cancer nomograms: an update. Eur Urol 2006;50:914-926.

9. Blumberg JW. PDA applications for physicians. ASCO News 2004:16:S4-6.

10. Kattan MW, Scardino PT. Evidence for the usefulness of nomograms. Nat Clin Pract Urol 2007:4:638-639.

11. Thompson IM, Ankerst DP, Chi C, et al. Assessing prostate cancer risk: results from the prostate cancer prevention trial. J Natl Cancer Inst 2006;98:529-534.

12. Kranse R, Beemsterboer P, Rietbergen $K$, et al. Predictors for biopsy outcome in the European Randomized Study of Screening for Prostate Cancer (Rotterdam region). Prostate 1999;39:316-322.

13. Thompson IM, Ankerst DP, Chi C, et al. Operating characteristics of prostate-specific antigen in men with an initial PSA level of 3.0 $\mathrm{ng} / \mathrm{ml}$ or lower. JAMA 2005;294:33-70.

14. Ankerst DP, Groskopf J, Day JR, et al. Predicting prostate cancer risk through incorporation of prostate cancer gene 3. J Urol 2008;180:1303-1308.

15. Parekh DJ, Ankerst DP, Higgins BA, et al. External validation of the prostate cancer prevention trial risk calculator in a screened population. Urology 2006;68:1152-1155.

16. Munekado KM, Yoshio N, Waturu I, et al. The American urological association symptom index for benign prostatic hyperplasia as a function of age, volume and ultrasonic appearance of the prostate. J Urol 1997;157:2160-2165.

17. Garzotto M, Hudson RG, Peters L, et al. Predictive modeling for the presence of prostate carcinoma using clinical laboratory, and ultrasound parameters in patients with prostate specific antigen levels $\leq 10 \mathrm{ng} / \mathrm{mL}$. Cancer 2003;98:1417-1422.

18. Nam RK, Toi A, Klotz LH, et al. Assessing individual risk for prostate cancer. J Clin Oncol 2007;25:3582-3588.

19. Schroder F, Kattan MW. The comparability of models for predicting the risk of a positive prostate biopsy with prostate-specific antigen alone; a systematic review. Eur Urol 2008;54:274-290.

20. Kaiser J. Researcher, two universities sued over validity of prostate cancer test. Science 2009;325:1484.

21. Zheng SL, Sun J, Wiklund F, et al. Cumulative association of five genetic variants with prostate cancer. N Engl J Med 2008:358:910_ 919.

22. Haas GP, Delongchamps NB, Jones RF, et al. Needle biopsies on autopsy prostates: sensitivity of cancer detection based on true prevalence. J Natl Cancer Inst 2007;99:1484-1489. 